\title{
Impact of Septoplasty on Mean Platelet Volume Levels in Patients with Nasal Septal Deviation
}

\author{
Prof. Dr. A.R. Ali Sulthan MS., DLO., ${ }^{1}$ Prof. Dr. V. Saravanan MS ${ }^{2}$, \\ Dr.Anish karthick ${ }^{3}$ \\ (Professor, ${ }^{I}$ Department Of ENT, Coimbatore Medical College and Hospital, Coimbatore, India.) \\ (Professor, ${ }^{2}$ Department Of ENT, Coimbatore Medical College and Hospital, Coimbatore, India.) \\ (Junior resident, ${ }^{2}$ Department Of ENT, Coimbatore Medical College and Hospital, Coimbatore, India.)
}

\begin{abstract}
:
Objective: Septal deviation being one of the common nasal pathology has strong association with chronic alveolar hypoxia. Mean Platelet Volume (MPV) is a widely used marker for chronic hypoxia. This study aims at establishing a relation between septal deviation and Mean Platelet Volume (MPV) and its effect on surgical correction of deviated septum.

Materials and Methods: Ninety-eight patients with symptomatic nasal septal deviation were enrolled for the study and operated. Pre-operative and post-operative Mean Platelet Volume (MPV) was noted .Comparative multivariate analyses between indicator factors and septal deviation were conducted.

Results: The pre-operative Mean Platelet Volume (MPV) levels of the patients who underwent septal surgery were significantly higher than the post operative Mean Platelet Volume (MPV) levels.

Conclusion: As a conclusion, Mean Platelet Volume (MPV) may be a helpful marker for ENT surgeons to evaluate Deviated Nasal Septum, which may cause chronic hypoxic complication.
\end{abstract}

Keywords: Airway obstruction, Deviated nasal septum (DNS), Hypoxia, Mean Platelet Volume (MPV).

\section{Introduction}

Deviated nasal septum causing chronic fixed nasal obstruction is one of the most common nasal pathology [1]. Yet, only a fraction of the population with deviation is symptomatic, as in when there is a sharp spur impinging on turbinate or the deviation blocks ostium-meatal complex (OMC) then patient might have head ache and facial pain. A persistent fixed upper airway obstruction can adversely affect lower airway and can lead to prolonged alveolar hypoxia, pulmonary hypertension, COPD, cardiac disease and vascular complications, also worsen obstructive sleep apnea and sleep-related breathing disorder [2]. There is no single mode to measure the impact of deviated septum on such systemic complications possibly induced by chronic alveolar hypoxia. Also, there are no precise criteria other than symptomatic nasal obstruction for surgical correction of septal deviation.

Mean Platelet volume (MPV) is a platelet activation marker in chronic hypoxia. . Mean Platelet volume (MPV) is a parameter in routine blood count and is a most widely used surrogate markers of platelet function [3][4]. It is proved that MPV increase in conditions like adenoid hypertrophy, pulmonary hypertension and atherosclerosis. There are studies showing MPV as a marker for atherosclerosis.

There are studies relating Mean Platelet Volume and Acoustic Rhinometry (nasal resistance) with no relevance to surgical outcome [5]. Acoustic Rhinometry is available only in few centers and is cumbersome.

This study aims at establishing a relationship between septal deviation and mean platelet volume (MPV) and impact of septal correction on MPV, thereby providing a marker for septal deviation induced lower airway complications and a guideline for septal surgery.

\section{Materials And Methodology}

Our study was approved by the local ethics committee and conducted in accordance with the ethical principles. Written informed consent form was obtained from all participants before the study.

Ninety-eight patients above 15 years of age and below 55 years of age with symptomatic nasal septal deviation for more than 6 months at Government Coimbatore medical College and hospital were enrolled for the study from March 2016 to March 2017.

Patients with concurrent nasal pathology and other causes of nasal obstruction (polyp, mass, AFRS), nasopharyngeal pathology/OSA syndrome, palatal pathology, chronic drug intake including non-steroidal antiinflammatory drugs/ corticosteroids, systemic disease such as any known cardiac diseases, Hypertension, CAD, disease that potentially affect blood pressure, any arrhythmias, or lung disease like COPD, diabetes mellitus, 
chronic renal or hepatic disease cancer, Hypercholesterolemia, obesity and bleeding diathesis and any infection autoimmune disease were excluded.

Patients enrolled to study with complaints of nasal obstruction and head ache, were thoroughly evaluated, detailed history, complete clinical examination including vitals recorded and examined for any other primary ENT pathology. After ruling-out other co-morbidities and primary ENT pathology, patient is evaluated with radiological investigation and basic baseline blood investigations.

CT Paranasal sinuses (coronal, axial and sagittal cuts) were taken. The point of maximum deviation of septum and the degree of deviation from midline in millimeters $(\mathrm{mm})$ were noted in Coronal and axial cuts (as in Fig.1 and Fig 2). Type of deviation noted (right, left, s-shaped, presence of spur).

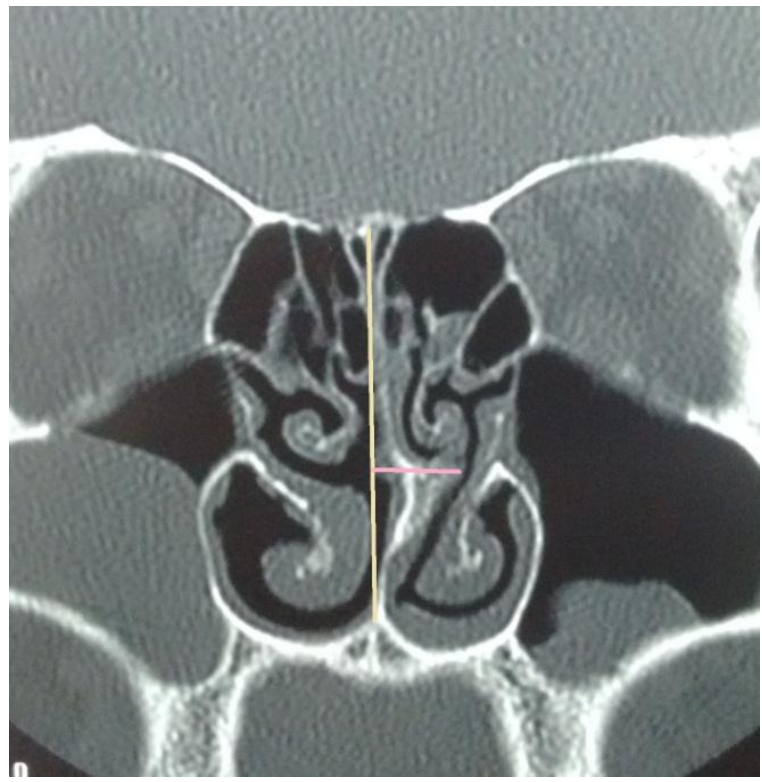

Fig.1 Coronal cut of CT Paranasal Sinus, cut with maximum deviation, yellow line represents midline, pink line shows degree of septal deviation.

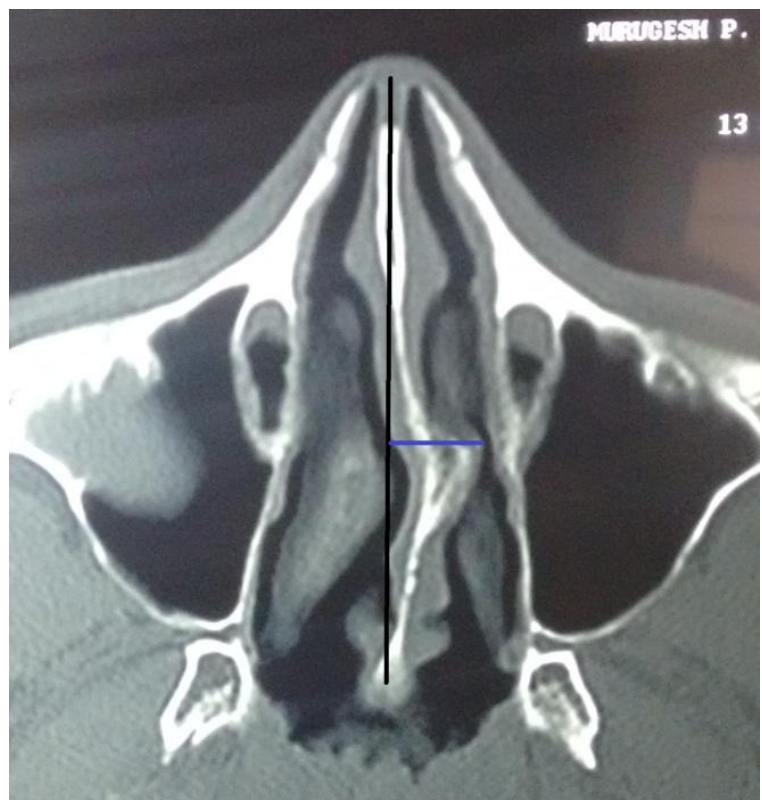

Fig.2 Axial cut of CT Paranasal sinus, cut corresponding to maximum deviation, black line represents midline, blue line shows degree of deviation

Complete blood hemogram with bleeding time and clotting time done. Mean Platelet Volume were noted down in all patients. Urine routine and microscopy, Random blood sugar estimation, renal function test, ECG, Chest $\mathrm{X}$ ray were done. 
After obtaining anesthetic fitness the patients were posted for surgery. Under general anesthesia patient underwent septal correction by means of SMR/Septoplasty. Surgery done in routine manner and adequate correction obtained.

Post operative complete blood count taken and Mean Platelet Volume were noted at one week after surgery, a month after surgery and after 6 months.

Statistical analysis of pre-operative MPV (patient and controls), degree of septal deviation and post operative MPV were done using Two sample T-test. $p$ value was calculated to determine if any significant relationship exists between these variables.

\section{Results}

Present study includes 98 patients, out of which, 50 patients $(51.02 \%)$ were males while 48 patients $(48.98 \%)$ were females. Male to female ratio being 1.04:1.

In the present study maximum number of patients, 37 patients $(37.76 \%)$ were reported in the age group of 16-25 years, male patients in this group were $20(20.41 \%)$ and female patients were $17(17.35 \%)$, followed by age group 26-35 years with total of 33 patients (33.67\%), male patients in this group were $19(19.39 \%)$ and female patients were $14(14.29 \%)$, followed by age group 36-45 years with 19 patients (19.39\%), male patients in this group were $9(9.18 \%)$ and female patients were $10(10.20 \%)$, and least number of patients in age group 46-55 years with 9 patients $(9.18 \%)$, male patients in this group were $2(2.04 \%)$ and female patients were 7 (7.14\%). The association between MPV and increasing age showed no significant difference. (See in Table 1)

Table 1

\begin{tabular}{|l|l|l|l|l|l|l|l|}
\hline \multicolumn{2}{|l|}{ variable } & Male & MPV(fl) & Female & MPV(fl) & Total & MPV(fl) \\
\cline { 3 - 8 } \multicolumn{2}{|l|}{} & 50 & $11.09 \pm 0.59$ & 48 & $11.13 \pm 0.59$ & 98 & $11.11 \pm 0.59$ \\
\hline \multirow{3}{*}{ Age } & $16-25$ & 20 & $11.06 \pm 0.69$ & 17 & $11.13 \pm 058$ & 37 & $13.14 \pm 1.72$ \\
\cline { 2 - 8 } & $26-35$ & 19 & $11.12 \pm 0.56$ & 14 & $11.16 \pm 0.71$ & 33 & $12.96 \pm 1.48$ \\
\cline { 2 - 8 } & $36-45$ & 9 & $11.14 \pm 0.53$ & 10 & $11.05 \pm 0.55$ & 19 & $12.26 \pm 1.53$ \\
\cline { 2 - 8 } & $46-55$ & 2 & $10.85 \pm 0.35$ & 7 & $11.19 \pm 0.45$ & 9 & $11.79 \pm 1.16$ \\
\hline
\end{tabular}

The degree of deviation of the septum and its association with pre-operative and post-operative MPV were studied. The maximum septal deviation of patients in study group was $16 \mathrm{~mm}$. so comparison was made between less than $8 \mathrm{~mm}$ ( 45 patients) deviation and more than $8 \mathrm{~mm}$ (53 patients) deviation. In less than $8 \mathrm{~mm}$ group we had a pre-operative MPV of $10.89 \pm 1.1$ and in more than $8 \mathrm{~mm}$ group we had a pre-operative MPV of $11.29 \pm 0.94$. There was a statistically significant reduction of MPV in the more than $8 \mathrm{~mm}$ of deviation group.

In this study, 41 patients $(41.84 \%)$ had right sided deviation, of which $22(22.45 \%)$ were males and 19 (19.39\%) were females, 34 patients $(34.69 \%)$ had spur. Also 46 patients $(46.94 \%)$ had left sided deviation, of which $21(21.43 \%)$ were males and $25(25.51 \%)$ were females, 36 patients $(36.73 \%)$ had spur. Only 11 patients $(11.22 \%)$ had S-Shaped deviation, of which 7(7.14\%) were males and $4(4.08 \%)$ were females, 9 patients $(9.18 \%)$ had spur. MPV was maximum in S-Shaped deviation group $(11.45 \pm 1.04)$ and was almost equal in right $(11.02 \pm 1.10)$ and left $(11.04 \pm 1.02)$ deviation groups preoperatively. There was marked reduction of MPV noted in S-Shaped deviation group $(7.83 \pm 0.98) 6$ months post-operatively, compared to right $(8.25 \pm$ $1.01)$ and left $(8.21 \pm 0.99)$ groups. Also, there was a significant reduction of MPV post-operatively in patients with septal spur (pre-operative MPV $-11.25 \pm 1.12$, post-operative MPV at 6 months $7.91 \pm 1.18$ ). (see in Table 2)

Table 2

\begin{tabular}{c|c|c|c|c}
\hline \multicolumn{2}{c|}{ Variables } & Total & Male & Female \\
\hline \multirow{3}{*}{$\begin{array}{c}\text { Type of } \\
\text { deviation }\end{array}$} & Right & $41(41.84 \%)$ & $22(22.45 \%)$ & $19(19.39 \%)$ \\
\cline { 2 - 5 } & Left & $46(46.94 \%)$ & $21(21.43 \%)$ & $25(25.51 \%)$ \\
\cline { 2 - 5 } & S-shaped & $11(11.22 \%)$ & $7(7.14 \%)$ & $4(4.08 \%)$ \\
\cline { 2 - 5 } & spur & $79(80.61 \%)$ & $38(38.78 \%)$ & $4141.84 \%)$ \\
\hline \multicolumn{2}{c}{ Hemoglobin(gms\%) } & $12.8 \pm 1.604$ & $13.92 \pm 1.26$ & $11.60 \pm 0.918$ \\
\hline \multicolumn{2}{c}{ RBC Count } & $4.73 \pm 0.595$ & $4.99 \pm 0.613$ & $4.46 \pm 0.435$ \\
\hline \multicolumn{2}{c}{ Platelet Count } & $3.08 \pm 1.117$ & $2.98 \pm 1.155$ & $3.18 \pm 1.08$ \\
\hline
\end{tabular}

The pre- operative MPV for the total patients who underwent septal surgery was $11.11 \pm 0.586$, of which male patients had a pre-operative MPV of $11.09 \pm 0.592$ and female patients had a pre-operative MPV of $11.13 \pm 0.585$. The post-operative first week MPV of all patients were $9.726 \pm 0.732$, of which male patients had MPV of $9.67 \pm 0.67$ and female patients had MPV of $9.79 \pm 0.777$. The post-operative first month MPV of all patients were $8.51 \pm 0.575$, of which male patients had MPV of $8.51 \pm 0.593$ and female patients had MPV of 
$8.51 \pm 0.561$. The post-operative 6 month MPV of all patients were $8.201 \pm 0.531$, of which male patients had MPV of $8.18 \pm 0.541$ and female patients had MPV of $8.23 \pm 0.525$. (See in Table 3)

Table 3

\begin{tabular}{|c|c|c|c|c|c|}
\hline Variables & Pre-operative & \multicolumn{2}{|c|}{ Post-operative MPV(fl) } & \multirow{2}{*}{$\begin{array}{c}\text { S.E } \\
0.0947\end{array}$} & \multirow{2}{*}{$\begin{array}{l}\text { P value } \\
<0.001 \\
\end{array}$} \\
\hline \multirow{3}{*}{ Total } & \multirow{3}{*}{$11.11 \pm 0.586$} & Week 1 & $9.72 \pm 0.732$ & & \\
\hline & & Month 1 & $8.51 \pm 0.575$ & 0.0829 & $<0.001$ \\
\hline & & Month 6 & $8.201 \pm 0.531$ & 0.0799 & $<0.001$ \\
\hline \multirow{3}{*}{ Male } & \multirow{3}{*}{$11.09 \pm 0.592$} & Week 1 & $9.67 \pm 0.69$ & 0.129 & $<0.001$ \\
\hline & & Month 1 & $8.51 \pm 0.593$ & 0.119 & $<0.001$ \\
\hline & & Month 6 & $8.18 \pm 0.541$ & 0.113 & $<0.001$ \\
\hline \multirow{3}{*}{ Female } & \multirow{3}{*}{$11.13 \pm 0.585$} & Week 1 & $9.79 \pm 0.777$ & 0.14 & $<0.001$ \\
\hline & & Month 1 & $8.51 \pm 0.561$ & 0.117 & $<0.001$ \\
\hline & & Month 6 & $8.23 \pm 0.525$ & 0.113 & $<0.001$ \\
\hline
\end{tabular}

It was observed that the post-operative MPV markedly reduced with statistically significant levels than the preoperative MPV of all patients.

The MPV values in accordance with age and sex were also studied but the observations were not statistically significant for age or between genders pre- operatively and post operatively.

\section{Discussion}

Deviated nasal septum is one of the commonly faced clinical diagnoses in patients presenting with nasal complaints to ENT out-patient department. Most patients might only have a mild nasal block, many a time it's just an incidental finding, but the spectrum might vary from asymptomatic septal deviation to an impinging or obstructing spur with unbearable head ache and at times spontaneous profuse bleeding from nose. Though at nasal level its asymptomatic, septal deviation can produce chronic alveolar hypoventilation and subsequently can lead to hypoxia. Chronic hypoxia thus produced stimulates IL-6 causing platelet activation and can lead to changes in platelet structure and function [6]. This adversely affects the platelet indices like Platelet Distribution Width (PDW) and Mean Platelet Volume (MPV). MPV is a novel and potential indicator for platelet function, in literature there are several articles regarding association of MPV to CAD and atherosclerosis, implying inflammatory,cardiac and thrombotic risk with platelet dysfunction[6]. Limited number of studies exists regarding MPV and DNS relationship.

The type of deviation with pre-operative and post-operative MPV had a positive relation in our study; the preoperative MPV was higher in patients with S-Shaped deviation and the post-operative $6^{\text {th }}$ month MPV had drastically reduced in this group. Also, the degree of deviation of the septum and its association with preoperative and post-operative MPV were studied. The maximum septal deviation of patients in study group was $16 \mathrm{~mm}$. so comparison was made between less than $8 \mathrm{~mm}$ (45 patients) deviation and more than $8 \mathrm{~mm}$ (53 patients) deviation. In less than $8 \mathrm{~mm}$ group we had a pre-operative MPV of $10.89 \pm 1.1$ and in more than $8 \mathrm{~mm}$ group we had a pre-operative MPV of $11.29 \pm 0.94$. There was a statistically significant $(<0.05)$ reduction of MPV in the more than $8 \mathrm{~mm}$ of deviation group. This correlates with the study proposed by Jin HR et al. owing to severity of airway obstruction caused by S-shaped deviation and septal deviation more than $8 \mathrm{~mm}$ from midline.

In this study, the pre-operative MPV of the patients were found to be high, similar to the studies by Ulu et al.[5] and Sagit et al.[7] owing to the chronic nasal obstruction induced hypoxia.

In this study the post operative MPV were taken at 1 week, 1 month and 6 months after surgery, these mean MPV values at 1week, 1 month and 6 months after surgery were compared with the pre operative mean MPV individually and it was found that all three post-operative mean MPV had reduced drastically with a statistical significance of $<0.001$ with that of the pre-operative MPV. Similar results were obtained with studies conducted by VarolE et al.[8] which shows that septal correction can relieve the upper aiway obstruction which in turn can alter platelet function.

There was no age or gender associated relationship found between the study populations MPV, preoperatively and post operatively. Also there were no significant relationships noted between other variables in the study with MPV like hemoglobin level, Red Blood Cell count or platelet count.

\section{Conclusion}

The present study reveals that chronic hypoxia caused by septal deviation alters platelet function, which in turn was shown by increase in preoperative MPV of all patients in this study and MPV levels reduces following septal surgery (with a statistical significance) indicates that this marker can be used as one of the baseline indicator for septal surgery, yet ENT surgeons should not overlook MPV values while evaluating 
patients because it might speed up operation decision to prevent the complications. Study proves that MPV has a correlation with deviated nasal septum yet the exact mechanism by which it affects is in shadows to explore.

\section{References}

[1]. Sipila J, Suonpaa J. A prospective study using rhinomanometry and patient clinical satisfaction to determine if objective measurements of nasal airway resistance can improve the quality of septoplasty. Eur Arch Otorhinolaryngol. 1997;254(8):38790 .

[2]. Sagit M, Korkmaz F, Kavugudurmaz M, Somdas MA. Impact of septoplasty on mean platelet volume levels in patients with marked nasal septal deviation. J Craniofac Surg 2012; 23(4):974-976.

[3]. Kapsoritakis AN, Koukourakis MI, Sfiridaki A, Potamianos SP, Kosmadaki MG, Koutroubakis IE, et al. Mean platelet volume: a useful marker of inflammatory bowel disease activity. Am J Gastroenterol 2001; 96(3): 776-781

[4]. Kisacik B, Tufan A, Kalyoncu U, Karadag O, Akdogan A, Ozturk MA, et al. Mean platelet volume (MPV) as an inflammatory marker in

[5]. ankylosing spondylitis and rheumatoid arthritis. Joint Bone Spine 2008; 75(3):291-294.

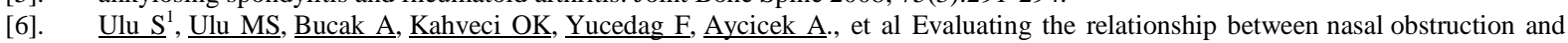
mean platelet volume by using acoustic rhinometry in patients with septum deviation. Rhinology. 2013 Sep;51(3):249-52

[7]. Şahin Ulu et al. Mean Platelet Volume as a Potential Marker for Predicting Hypoxia in Children with Adenotonsillar Hypertrophy. Klinik Çalışma. Van Tıp Dergisi: 22(1): 10-13, 2015

[8]. Sagit M et al.(2012) Septoplasty decreases the Mean Platelet Volume levels in patients with marked septal deviations. J Craniofac Surg 23(4):974-976. doi:10.1097/SCS.0b013e31824e2c08

[9]. Varol E, Dogan A, Erdogan D, Ozaydin M, Peker RO. Septoplasty decreases mean platelet volume levels in patients with marked nasal septal deviation. J Craniofac Surg. 2012 Nov;23(6):1938. doi: 10.1097/SCS.0b013e318271021c. 